\title{
ELECTRICAL STUDIES IN THE DIAGNOSIS OF COMPRESSION OF THE LUMBAR ROOT
}

\author{
ANTHONY LEYSHON, ERNEST O' G. KIRWAN, CHRISTOPHER B. WYNN PARRY \\ From the Royal National Orthopaedic Hospital, London
}

\begin{abstract}
A series of 100 patients with pain in the leg was studied and the accuracy of electrical studies in the diagnosis of nerve root lesions was investigated before operation. The electrical studies which were performed on all the patients, included recordings of fibrillation potentials, H-reflex and ankle reflex latencies. This diagnostic technique was found to be more accurate than radiculography or clinical examination and did not give false evidence. Seventy patients were classed as having a lesion of the nerve root. Fifty of these patients were treated surgically. The operation revealed compression of the nerve root by osteophytes arising from degenerative and incompletely dislocated posterior facet joints in $\mathbf{4 3}$ patients.
\end{abstract}

The pitfalls of inaccurate diagnosis are most apparent in those patients with pain in the leg associated with a disorder of the lumbar spine. A correct diagnosis is essential to avoid unnecessary, useless or harmful treatment. The many causes of sciatica have been enumerated by Macnab (1977) and include radicular pain. Traditionally, the diagnosis of pain in the leg due to nerve root dysfunction has relied upon clinical examination followed, where indicated, by contrast radiography of the lumbar spine. Clinical examination is unreliable in the diagnosis of pain in the leg caused by a prolapsed intervertebral disc and has an accuracy of 39 per cent (Lansche and Ford 1960). Edgar and Park (1974) showed that the straight leg raising test was positive in only 80 per cent of their patients with a prolapsed intervertebral disc diagnosed at operation. They were able to localise the level of the lesion in only 50 per cent by neurological examination. Physical signs such as tenderness at motor points in the lower limb muscles described by Gunn and Milbrandt (1976) may give additional information, useful in the diagnosis.

Contrast radiography, either myelography (Lansche and Ford 1960), radiculography (Salenius and Laurent 1977; Cook and Wise 1979), or epidural venography (Gargano, Meyer and Sheldon 1974) is a well established technique used to investigate prolapsed intervertebral discs. However, the interpretation of contrast radiographs performed after previous operation is notoriously difficult (Castan et al. 1977). These investigations are often unpleasant for the patient and may have both early and late complications. Selective infiltration of the nerve root with local anaesthetic is another useful diagnostic tool (Macnab 1971; Schutz et al. 1973; Krempen and Smith 1974), but this technique is also unpleasant for the patient.

Diagnosis is most difficult in patients with a long history of pain in the leg and in those who have had several previous operations on the back (Coventry and Stauffer 1969). Certain groups of patients who were referred to our unit were particularly difficult to assess. These included patients with previous operations or extensive arachnoiditis and those with normal results after neurological examination and radiculography. The accuracy of both clinical and radiological diagnosis was disappointing in these cases so electrical studies were tried as a diagnostic aid. The use of electromyography to investigate the compression of nerve roots is not new (Shea, Woods and Werden 1950). The majority of previous studies were performed on patients with pain in the leg caused by a prolapsed intervertebral disc (Bonner and Schmidt 1957; Marinacci 1958; Knutsson 1961; LaJoie 1972). The electromyographic diagnosis of recurrent radiculopathy after operation has been a particularly controversial subject and therefore has not been favoured by orthopaedic surgeons, especially in this country. Often electromyographic diagnosis of the root dysfunction has relied on the detection of electrical changes in the paraspinal muscles which are supplied segmentally by the posterior primary rami of the spinal nerves. Hence, denervation in these muscles found after operation has been interpreted as recurrent radiculopathy. See and Kraft (1975) have shown that after laminectomy, denervation may occur at several levels in the paraspinal muscles for at least 41 months
A. Leyshon, FRCS, Senior Orthopaedic Registrar

E. O' G. Kirwan, FRCS, Consultant Orthopaedic Surgeon

C. B. Wynn Parry, $M B E$, FRCP, FRCS, Consultant Rheumatologist

Requests for reprints should be sent to Mr A. Leyshon.
Royal National Orthopaedic Hospital.

234 Great Portland Street.

London W1N 6AD, England. 
after the operation without recurrent radicular symptoms. The use of paraspinal muscle electromyography in such cases is contra-indicated.

Confusion also arises if conduction velocities in the distal nerve segments are measured and used to detect proximal disturbance at the level of the nerve root, since these velocities are normal in patients with radiculopathy (Eisen, Schomer and Melmed 1977). Detection of muscle denervation in the lower limb is non-specific, but it may suggest a lesion of the nerve root (Knutsson 1961). Specific involvement of the nerve root may be indicated by increased latencies of the knee and ankle reflexes. The reflex times are measured electronically with a triggering patella-hammer (Malcolm 1951). Similarly, the H-reflex is mediated through the root of the first sacral nerve and increased $\mathrm{H}$-reflex latencies imply dysfunction of the root (Deschuytere and Rosselle $1970 ; 1973)$.

The segmental innervation of muscles proposed by Marinacci (1955), enables the dysfunction of a specific nerve root to be established from given patterns of denervation in the muscles of the lower limb (Table I). It was therefore decided to evaluate the role of electrical studies in the diagnosis of pain in the leg using three criteria: the presence and the patterns of muscle denervation, the increase in the $\mathrm{H}$-reflex latencies and the increase in the tendon-reflex latencies.

\section{CLINICAL MATERIAL AND METHODS}

One hundred consecutive patients referred to the problem-back unit of the Royal National Orthopaedic Hospital, London, were studied. There were 42 men and 58 women aged between 19 and 63 years (mean 39.8 years). Ninety-three patients complained of backache with a mean duration of seven years $(0.5-30.0$ years $)$, but all the patients in this group had pain in the leg with a mean duration of 5.4 years $(0.5-20$ years). The pain in the leg was unilateral in 91 patients (on the right side in 38 and on the left side in 53) and in nine patients it was bilateral. Fifty-seven patients had previously undergone myelographic or radiculographic examination and $\mathbf{4 8}$ had undergone spinal operations.

An extensive clinical history was obtained for each patient and a careful general and neurological examination performed, particular attention being paid to the following points: the presence of a scoliosis or list; the degree of spinal mobility; the straight leg raising test; deep tendon reflexes; the plantar response; wasting of muscle; tenderness of the muscle at motor points; weakness of the muscle and sensory deficit. Radiculography was performed in 66 patients using the water-soluble contrast medium methyl glucamine iocarmate (Dimer $\mathrm{X}$ ) or metrizamide (Amipaque). Standard lumbar radiculography was performed under fluoroscopic control and the results interpreted by experienced radiologists. There were no technical failures in this series.

Electrical studies, using a Medelec MS6 electrophysiological recording unit, were performed on all patients. The electrical responses obtained were displayed on an oscilloscope and recorded on paper. Concentric needle electrodes were used to sample individual muscles of the lower limbs in at least nine different sites. Denervation was reported if characteristic fibrillation potentials or positive sharp waves persisted for at least a minute and could be detected in at least three sampling sites. The interference pattern during maximal voluntary effort was then examined with the aid of a cathode ray oscilloscope and the amount of motor-unit activity estimated. This was recorded as either a discrete, partial or complete interference pattern.
Table I. Segmental innervation for muscles of the lower limb (modified from Marinacci 1955)

\begin{tabular}{|l|c|}
\hline \multicolumn{1}{|c|}{ Muscle innervated } & Nerve \\
\hline Quadriceps femoris & L4 \\
\hline $\begin{array}{l}\text { Tibialis anterior } \\
\text { Extensor hallucis longus } \\
\text { Gastrocnemius (lateral head) } \\
\text { Extensor digitorum brevis }\end{array}$ & L5 \\
\hline $\begin{array}{l}\text { Soleus } \\
\text { Gastrocnemius (medial head) } \\
\text { Abductor hallucis }\end{array}$ & $\mathrm{S} 1$ \\
\hline
\end{tabular}

Any increase in the polyphasicity of the motor-unit potentials was ignored, but the detection of giant units was considered important. One unit was termed a giant unit, and was indicative of chronic partial denervation, if the amplitude of the potential was eight millivolts or more in any of the muscles sampled.

$\mathrm{H}$-reflex latencies were measured on the right and left sides using the technique described by Braddom and Johnson (1974). An increase in the latency of more than two milliseconds was considered indicative of a lesion in the root of the S1 nerve. The latencies of the ankle reflex were measured using an electronic hammer which triggered the time base of the electromyograph (Malcolm 1951). Recordings were made with a surface electrode placed over the medial head of the gastrocnemius. A comparison was made between the latencies on the two sides, but no attention was paid to the amplitude of the response. A difference of more than two milliseconds between the latencies was considered significant enough to indicate a slowing across the root of the S1 nerve.

If there was the slightest doubt in any patient as to whether the condition might be a peripheral neuropathy, the sensory action potential of the sural nerve was measured. This was carried out using the standard technique, stimulating the sural nerve antidromically in the lower-third of the calf slightly lateral to the mid-line and recording with surface electrodes along the nerve below the lateral malleolus. The motor conduction velocity was also measured in the lateral and medial popliteal nerves in these patients. Recording was made over the extensor digitorum brevis and the abductor hallucis muscles respectively and stimulation of the respective nerves at the knee and ankle. Sural nerve sensory action potentials and motor conduction velocities were found to be normal in all the patients investigated.

\section{RESULTS}

The 100 patients in this study were categorised using the available information into two groups: 70 patients with pain from the nerve root and 30 without such pain. Twenty of the 70 patients with positive evidence of radicular pain, but with insufficient symptoms to justify surgery, intercurrent disease, poor psychometrics or with backache as the major symptom, were treated conservatively. The remaining 50 patients were representative of the whole series with respect to their age, sex and the duration of symptoms. They included 11 patients who had undergone previous myelography or radiculography alone and 22 patients who had previously undergone surgical treatment for pain in the leg 
Table II. The nerve roots involved in the group of 50 patients with evidence of radicular pain

\begin{tabular}{|c|c|c|}
\hline Root involved & Right & Left \\
\hline L4 & 0 & 1 \\
L5 & 11 & 21 \\
S1 & 12 & 15 \\
\hline
\end{tabular}

Table III. The types of abnormal nerve roots found in the 50 patients treated surgically

\begin{tabular}{|l|c|c|c|}
\hline & & \multicolumn{2}{|c|}{ No previous surgery } \\
\cline { 3 - 4 } Type of root & Previous surgery & Myelography & No myelography \\
\hline Fibrotic & 14 & 8 & 9 \\
Swollen & 8 & 3 & 8 \\
\hline
\end{tabular}

Table IV. The relative accuracy of the three diagnostic methods in determining the presence of a nerve root lesion and the specific root involved

\begin{tabular}{|l|c|c|c|c|c|}
\hline \multirow{2}{*}{ Diagnostic method } & \multirow{2}{*}{ Number of patients } & \multicolumn{2}{|c|}{ Correct diagnosis of nerve root lesion } & \multicolumn{2}{c|}{ Correct diagnosis of specific root } \\
\cline { 3 - 6 } & Number of patients & Accuracy (per cent) & Number of patients & Accuracy (per cent) \\
\hline Clinical & 50 & 20 & 40 & 12 & 24 \\
Radiculographic & 46 & 24 & 52 & 15 & 33 \\
Electrical & 50 & 45 & 90 & 38 & 76 \\
\hline
\end{tabular}

preceded by contrast radiography. These 50 patients were treated surgically.

The nerve root which had been implicated before operation was exposed and where indicated this was followed by exposure of the more proximal or distal root. Pathological nerve roots were found in all 50 patients (Table II). Two roots were involved on the same side in seven patients and bilaterally in three patients. The overall appearance of the nerve root involved was always one of two distinct types: fibrotic type when the root was hard, white, thin and fibrotic or swollen type when it was soft, pink, oedematous and particularly irritable to handling. The fibrotic type, which corresponds to the battered root described by Bertrand (1975), was found in 14 of the 22 patients who had undergone previous surgical treatment and in 17 of the remaining 28 patients who had had no such previous treatment (Table III).

Damage to the nerve roots which occurred in 43 patients ( 86 per cent) was caused by compression from osteophytes arising from degenerative and incompletely dislocated posterior facet joints, sometimes combined with kinking of the nerve root around the pedicle of the vertebral arch (Macnab 1971). This group included 11 patients with arachnoiditis in whom a single root was compressed extradurally by bone. No dural compression was present. Four of the remaining seven patients showed compression of the nerve root caused by sequestrated intervertebral disc material and two showed a combination of a sequestrated disc and osteophytic compression. In one patient the root was free without external compression, but there was well marked and localised fibrosis of the nerve-root sleeve. Evaluation of diagnostic methods. After the operation it was possible to compare the diagnostic accuracy of clinical examination, radiculography and electrical studies. Accuracy in diagnosing the presence of a lesion of the nerve root was only 40 per cent for the clinical examination, 52 per cent using radiculography and 90 per cent with the electrical studies.

Although the accuracy in diagnosing the specific root involved was lower for all three methods, the electrical studies were still the most accurate (Table IV). Clinical examination. In no patient was a scoliosis or list present. Spinal mobility was full in 21 patients, slightly restricted in 19 and very restricted in 10 patients. In restricted movement the lumbar spine was generally stiff with no particular abnormal rhythm of movement. The limitation usually applied equally to flexion, extension, lateral flexion and rotation. The straight leg raising test was negative in 24 patients and positive in 26 , although in this latter group there was no particular pattern of induced pain on straight leg raising such as that described by Edgar and Park (1974).

The ankle reflex was reduced in three patients, absent in 15, but demonstrated to be present and equal in 32 patients. The plantar response was always normal. Wasting of the muscles was observed in 21 patients and in five of these wasting was confined entirely to the extensor digitorum brevis muscle. There was tenderness at the motor points of the muscles of the lower limb in 10 patients, but this sign was only accurate in localising the specific nerve root involved in four. Weakness of the muscles was observed in 12 patients. This weakness was localised in the extensor hallucis longus muscle in nine patients in whom it was helpful and accurate in implicating involvement of the $\mathrm{L} 5$ nerve root. A sensory deficit which was tested by light touch and pin-pricks was present in 19 patients. This deficit was of a sufficiently clear dermatomal pattern to allow accurate 
localisation of the nerve root involved in only eight patients.

Radiculography. In the group of 50 patients treated surgically 46 had radiculograms. Twenty-two radiculograms (48 per cent) showed no abnormality. These investigations giving false negative results were more common in L5 lesions (12 patients) than S1 lesions ( 7 patients), whilst three patients had lesions of both the L5 and $S 1$ roots.

Twenty-four radiculograms were abnormal, but only 15 of these were interpreted as showing an unequivocal lesion of the root involved. Two patients with moderately extensive arachnoiditis and a single swollen nerve root suggesting a uniradicular lesion were included. The remaining nine radiculograms showed such extensive arachnoiditis with blunting or complete absence of multiple roots that individual affected roots could not be identified.

Electrical studies. Evidence of root lesion was found in 45 patients using these studies. The diagnosis was supported in 40 patients by increases in the $\mathrm{H}$-reflex latency or in the ankle reflex latency and from evidence of denervation. In five patients, who were subsequently found to have a lesion of only the $S 1$ roots, there were no signs of muscle denervation and diagnosis was based solely on abnormalities of the $\mathrm{H}$-reflex and ankle reflex latencies.

Spontaneous fibrillation was seen regardless of the duration of symptoms. Giant units were not observed in patients who had had radicular pain lasting less than 10 months. The five patients (10 per cent) with normal electrical studies were treated surgically on the basis of positive clinical and radiculographic evidence for dysfunction of the nerve root. In two of these patients with false negative results, sequestrated intervertebral disc tissue was the cause of dysfunction and in another two sequestrated disc tissue was an additional cause.

\section{DISCUSSION}

This study emphasises the difficulties in diagnosing radicular pain in a pre-selected group of patients with long histories of pain in the leg. The clinical history and physical signs may confuse the diagnosis. Signs of a lesion of the root commonly seen in patients with pain caused by a prolapsed intervertebral disc are rarely found. In this group there were few local signs in the lumbar spine and in only 52 per cent of the patients was the straight leg raising test positive. Clinical assessment of the ankle reflex was unreliable. Eighteen patients were found to have a reduced or absent ankle reflex using this method, but only 10 were confirmed to be abnormal with electrical testing of the ankle reflex latencies. Other clinical features could have suggested lesions of the S1 root in the eight patients with normal reflex latencies, but all were subsequently proven to have compression of the L5 nerve root.
Muscle wasting was a useful physical sign, if present, but in many patients it was not obvious and was only revealed on measurement. Wasting of the extensor digitorum brevis muscle which is mainly supplied by the L5 nerve (Kugelberg and Petersén 1950) was easily seen and was therefore particularly useful in determining the level of the root lesion. Muscle weakness was also a useful diagnostic sign, whereas mapping the sensory deficit was usually confusing.

The high percentage of normal radiculograms in this study reflects the site of compression of the root. In patients with a prolapsed intervertebral disc the root is compressed more centrally within the vertebral canal and therefore produces the characteristic radiculographic appearance of blunting of the nerve root. In the patients with compression caused by posterior facet osteophytes the lesion of the root is too lateral to be detected by radiculography. Electrical studies were more accurate in predicting the presence of a lesion than in determining the specific root involved. We believe that this latter inaccuracy is due to variation in some patients from the pattern of segmental innervation proposed by Marinacci (1955). A study at this hospital by Young (personal communication) involving intraoperative stimulation of the lumbar nerve roots and recording from the lower limb muscles confirmed Marinacci's findings but showed that the extensor digitorum brevis was innervated by the L5 nerve in 80 per cent and by the $S 1$ nerve in 20 per cent of the patients. Similarly the lateral head of the gastrocnemius was innervated by the L5 nerve in 75 per cent and the S1 nerve in 25 per cent of the patients studied.

Electrical studies do not enable us to predict those patients with a reversible lesion of the chronically compressed nerve root and therefore cannot be used to forecast the outcome of operation. Neither do we advocate surgical treatment for all patients with evidence of dysfunction of the root from such studies since clinical examination of the patient as a whole is essential. An important feature of this study was the absence of false evidence of a root lesion, which occurs with myelography or radiculography. In patients with chronic symptoms the root is almost certain to be compressed by bone and the intervertebral disc plays little or no part in the pathological process. This may explain the failure reported for disc surgery or chemonucleolysis in such patients (Graham 1975; Smith 1975; Salenius and Laurent 1977).

Eleven patients with radiculographic evidence of extensive arachnoiditis revealed physiological disturbance of a single root after electrical studies. At operation it was found that the arachnoiditis was not the cause of compression of the root and that in every case the compression was the result of osteophytes from the facet joints. The early results of operation in these patients were encouraging, 10 of them being free from pain in the leg at the follow-up made, on average, 16 
months later. The presence of arachnoiditis no longer prevents possible surgical treatment.

Patients who had been extensively investigated at other hospitals were included in this study, many of whom had been suspected of suffering from hysteria or depression, or were thought to be malingerers. Electrical studies help delineate these groups and allow appropriate treatment, either orthopaedic, psychiatric or rehabilitation to be instituted. We advocate the wider use of electrical studies in patients with possible radicular pain of the leg. The diagnostic use of electrical studies, together with an awareness of bone as a cause of nerve root compression should give better results in the treatment of these difficult problems.

\section{REFERENCES}

Bertrand G. The "battered" root problem. Orthop Clin North Am 1975;6:305-10.

Bonner FJ, Schmidt WH. Electromyography in disc disease. Arch Phys Med Rehabil 1957;38:689-91.

Braddom RI, Johnson EW. Standardization of H reflex and diagnostic use in S1 radiculopathy. Arch Phys Med Rehabil 1974;55:161-6.

Castan P, Bourbotte G, Herail JP, Maurel J. Follow-up and postoperative radiculography: a radiological analysis of 640 patients. J Neuroradiol 1977;4:49-93.

Cook PL, Wise K. A correlation of the surgical and radiculographic findings in lumbar disc herniation. Clin Radiol 1979;30:671-82.

Coventry MB, Stauffer RN. The multiply operated back. In: American Academy of Orthopaedic Surgeons. Symposium on the spine. St Louis: The CV Mosby Company, 1969: 132-42.

Deschuytere J, Rosselle N. Electromyographic and neurophysiological investigation in root compression syndromes in man. Electromyography 1970;10:339-40.

Deschuytere J, Roselle N. Diagnostic use of monosynaptic reflexes in L5 and S1 root compression. In: Desmedt JE, ed. New developments in electromyography and clinical neurophysiology. Basel: S. Karger, 1973;3:360-66.

Edgar MA, Park WM. Induced pain patterns on passive straight-leg raising in lower lumbar disc protrusion. J Bone Joint Surg [Br] 1974;56-B:658-67.

Eisen A, Schomer D, Melmed C. An electrophysiological method for examining lumbosacral root compression. Can J Neurol Sci 1977;4:117-23.

Gargano FP, Meyer JD, Sheldon JJ. Transfemoral ascending lumbar catheterization of the epidural veins in lumbar disk disease. Radiology 1974;111:329-36.

Graham CE. Chemonucleolysis. A preliminary report on a double blind study comparing chemonucleolysis and intradiscal administration of hydrocortisone in the treatment of backache and sciatica. Orthop Clin North Am 1975;6:259-63.

Gunn CC, Milbrandt WE. Tenderness at motor points. J Bone Joint Surg [Am] 1976;58-A:815-25.

Knutsson B. Comparative value of electromyographic, myelographic and clinical-neurological examinations in diagnosis of lumbar root compression syndrome. Acta Orthop Scand 1961; Suppl 49.

Krempen JF, Smith BS. Nerve-root injection. A method for evaluating the etiology of sciatica.J Bone Joint Surg [Am] 1974;56-A:1435-44.

Kugelberg E, Petersen I. Muscle weakness and wasting in sciatica due to fourth lumbar or lumbosacral disc herniations. $J$ Neurosurg 1950;7:270-7.

LaJoie WJ. Nerve root compression: correlation of electromyographic, myelographic and surgical findings. Arch Phys Med Rehabil 1972;53:390-2.

Lansche WE, Ford LT. Correlation of the myelogram with clinical and operative findings in lumbar disc lesions. J Bone Joint Surg [Am] 1960;42-A:193-206, 288.

Macnab I. Negative disc exploration. J Bone Joint Surg [Am] 1971;53-A:891-903.

Macnab I. Backache. Baltimore: Williams \& Wilkins Company, 1977.

Malcolm DS. A method of measuring reflex times applied in sciatica and other conditions due to nerve root compression. J Neurol Neurosurg Psychiatry $1951 ;$ NS14:15-24.

Marinacci AA. Clinical electromyography. Los Angeles: San Lucas Press, 1955.

Marinacci AA. The use of electromyography in the differential diagnosis of lumbar herniated disks. Bull Los Angeles Neurol Soc 1958;23:65-71.

Salenius P, Laurent LE. Results of operative treatment of lumbar disc herniation. A survey of 886 patients. Acta Orthop Scand 1977;48:630-4.

Schutz H, Lougheed WM, Wortzman G, Awerbuck B. Intervertebral nerve root block in the investigation of chronic lumbar disc disease. Can J Surg 1973;16:217-21.

See DH, Kratt GH. Electromyography in paraspinal muscles following surgery for root compression. Arch Phys Med Rehabil 1975;56:80-3.

Shen PA, Woods WW, Werden DM. Electromyography in diagnosis of nerve root compression syndrome. Arch Neurol 1950;64:93-104.

Smith L. Failures with chemonucleolysis. Orthop Clin North Am 1975;6:255-8. 